\title{
ÁCIDO MYRSINOICO A E DERIVADO: INIBIDORES DA FOTOSSÍNTESE IN VITRO
}

\section{Marcela Carmen de M. Burger, Gracielle S. de Oliveira e Antônio Carlos S. Menezes}

Unidade Universitária de Ciências Exatas e Tecnológicas, Universidade Estadual de Goiás - Br 153, 3105, 75132-903 Anápolis - GO, Brasil

Paulo Cezar Vieira e Maria Fátima das G. F. da Silva

Departamento de Química, Universidade Federal de São Carlos, Rod. Washington Luiz, km 235, 13565-905 São Carlos - SP, Brasil Thiago A. M. Veiga*

Departamento de Ciências Exatas e da Terra, Instituto de Ciências Ambientais, Químicas e Farmacêuticas, Universidade Federal de São Paulo, Rua Prof. Artur Riedel 275, 09972-270 Diadema - SP, Brasil

Recebido em 27/10/11; aceito em 14/2/12; publicado na web em 30/4/12

\begin{abstract}
MYRSINOIC A ACID AND ITS DERIVATIVE: IN VITRO INHIBITORS OF PHOTOSYNTHESIS. Myrsinoic A acid, isolated from Myrsine cuneifolia and its hydrogenated derivative had their effect on photosynthesis tested. The compounds inhibited the electron flow (basal, phosphorylating and uncoupled) from water to methylviologen; therefore, they act as Hill reaction inhibitors in spinach thylakoids. They inhibited partial reactions of PSII electron flow from water to 2,5-dichloro-1,4-benzoquinone, from water to sodium silicomolybdate, and partially electron flow from diphenylcarbazide to 2,6-dichloroindophenol. Their inhibition sites were at the donor and acceptor sides of PSII, between $\mathrm{P}_{680}$ and $\mathrm{Q}_{\mathrm{A}}$. Chlorophyll $a$ fluorescence measurements confirmed the behavior of the compounds (pool of quinones).
\end{abstract}

Keywords: Myrsine cuneifolia; myrsinoic A acid; photosynthesis.

\section{INTRODUÇÃO}

O Cerrado é um bioma de destaque mundial devido à alta biodiversidade e à ocorrência de espécies endêmicas, sendo o segundo maior bioma brasileiro, superado em área apenas pela Amazônia. Dentre as diversas famílias de plantas que compõem o Bioma Cerrado, encontra-se a família Myrsinaceae, que engloba cerca de 33 gêneros, com 1000 indivíduos largamente distribuídos pelas regiões tropicais do planeta. ${ }^{12}$

Vários compostos isolados de espécies do gênero Myrsine, família Myrsinaceae, foram descritos com atividades biológicas, tais como, rapanona, atividade bactericida. ${ }^{3}$ A citotoxidade de rapanona foi testada, e observou-se a atividade frente a quatro linhagens de células de: adenocarcinoma (HT-29), epitelioma (Hep-2), câncer de mama (MCF-7) e câncer de estômago (MKN-45). ${ }^{4}$

Através de uma breve revisão bibliográfica, verificou-se que a espécie Myrsine cuneifolia (Myrsinaceae) não possuía dados químicos publicados até o momento. Em função disto, bem como do grande potencial biológico apresentado por metabólitos produzidos pelo gênero e, ainda, pelo fácil acesso à planta, a mesma foi escolhida para estudo.

Os produtos naturais têm se mostrado como uma das fontes mais ricas e promissoras de novos compostos com atividade fitotóxica e reguladora do crescimento de plantas..$^{5-9}$ Contudo, o uso de produtos naturais como herbicidas, ou como modelos para conduzir programas de descoberta de herbicidas, é uma alternativa que não tem sido explorada tanto quanto foi para inseticidas e fungicidas. Produtos naturais fitotóxicos são, em geral, estruturalmente mais complexos que herbicidas sintéticos e não são economicamente obtidos por metodologias sintéticas tradicionais. Esta área está sendo explorada lentamente, ${ }^{10}$ talvez pelo pequeno número de pesquisadores envolvidos na área, pela falta de trabalhos em biotecnologia de produção de herbicidas naturais, ou mesmo, pela falta de ensaios que permitam conhecer o mecanismo de atividade dos compostos de interesse.

*e-mail: tveiga@unifesp.br
Nossos estudos sobre a interação de produtos naturais com os sítios da fotossíntese, visando a busca por modelos para herbicidas baseados em substâncias isoladas de plantas e micro-organismos, inicialmente mostraram-se muito interessantes. Os alcaloides flindersina e N-metil-flindersina se comportam de maneira semelhante ao herbicida comercial DCMU (3-(3,4-diclorofenil)-1,1-dimetiluréia). ${ }^{11} \mathrm{~A} \mathrm{~N}$-metil-flindersina apresenta ainda um segundo mecanismo de ação, pois interfere na oxidação da plastoquinona $\left(\mathrm{PQH}_{2}\right)$. O policetídeo, lasiodiplodina, possui três mecanismos de ação: inibidor da reação de Hill; inibe parcialmente o complexo enzimático que oxida a molécula de água e interage com o complexo proteico $\mathrm{CF}_{1}$ (centro catalítico do ATPase), através da inibição da atividade de $\mathrm{CF}_{1}-\mathrm{Mg}^{2+}$ - ATPase. ${ }^{12} \mathrm{~A}$ cumarina, siderina, também inibe a reação de Hill; inibe tanto o lado doador, quanto o lado aceptor de elétrons do FSII (Fotosistema II) entre $\mathrm{P}_{680}-\mathrm{Q}_{\mathrm{A}}$ (Centro reacional do FSII - Quinona A), criando os chamados centros silenciosos de reação, o que pode levar ao desenvolvimento de agentes fitotóxicos contra a fotossíntese de plantas daninhas. ${ }^{13}$ Dentro desse contexto, o metabólito isolado do extrato dos frutos de Myrsine cuneifolia e seu derivado foram submetidos a uma série de reações enzimáticas que auxiliaram no esclarecimento do (s) mecanismo (s) ação frente à fotossíntese.

\section{RESULTADOS E DISCUSSÃO}

\section{Efeito das substâncias 1 e 2 sobre o transporte de elétrons não} cíclico: reação de Hill

A formação de ATP pode ser inibida em qualquer uma das etapas da cadeia transportadora de elétrons. ${ }^{14}$ Dessa forma, para conhecer o efeito dos derivados de ácidos benzoicos sobre a produção de energia, foram investigados seus mecanismos de ação nas três etapas da cadeia de transporte de elétrons. Os resultados mostraram que a evolução de oxigênio em cloroplastos iluminados, tendo metilviologênio (MV) como aceptor de elétrons foi inibida na presença de ADP e fostato 
inorgânico (Pi). Observou-se que a inibição foi completamente realizada pela presença de $\mathrm{NH}_{4} \mathrm{Cl}$ como desacoplante (Figura 1).<smiles>CC(C)=CCC/C(C)=C/Cc1cc(C(=O)O)cc(CC=C(C)C)c1O</smiles><smiles>CC(C)CCCC(C)CCc1cc(C(=O)O)cc(CCC(C)C)c1O</smiles>

Figura 1. Estrutura química do ácido myrsinoico A (1) isolado de M. cuneifolia e seu derivado (2)

Os reagentes que bloqueiam o transporte de elétrons também inibem a síntese de ATP, a partir da formação do gradiente eletroquímico transmembrana. A força de saída para a síntese de ATP é dependente do fluxo de elétrons. Os compostos que aumentam a permeabilidade de prótons nas membranas de tilacoide desacoplam a fosforilação do fluxo de elétrons. Os agentes desacoplantes inibem a síntese de ATP pela diminuição do gradiente de prótons, porém o transporte de elétrons ainda pode ocorrer sob taxas elevadas. Em contraste, os inibidores diretos da fosforilação oxidativa bloqueiam tal fenômeno e, ainda, a porção do transporte de elétrons, que é consequência do fluxo de prótons ligado à fosforilação. ${ }^{15}$ Assim, a inibição descrita da fosforilação produzida pelas substâncias 1 e $\mathbf{2}$ pode ser explicada pelo seu efeito em cada uma das etapas da cadeia transportadora, através de reações de transferência de energia.

Avaliou-se o efeito dos compostos $\mathbf{1}$ e $\mathbf{2}$ sobre as razões de transporte de elétrons em diferentes condições (basal, fosforilante e desacoplado). Na ausência (basal) ou presença (fosforilante) de ADP, $\mathrm{Pi}$, ou $\mathrm{NH}_{4} \mathrm{Cl}$ (desacoplado) e usando $\mathrm{MV}$ como aceptor de elétrons, os compostos $\mathbf{1}$ e $\mathbf{2}$ inibiram a velocidade do transporte de elétrons em cloroplastos iluminados (Figuras 2 e 3). Observou-se que todas as condições do fluxo de elétrons foram inibidas pela substância $\mathbf{1}$, com valores de $\mathrm{I}_{50}$ de 32,0; 57,0 e 77,0 $\mu \mathrm{M}$ para os transportes basal, fosforilante e desacoplado, respectivamente. $O$ composto 2 apresentou comportamento similar, pois apresentou valores de $I_{50}$ de 25,0; 42,0 e $62,0 \mu \mathrm{M}$. A partir desses resultados foi possível concluir que ambos os compostos podem ser classificados como inibidores da reação de Hill.

\section{Localização dos ambientes de interação dos derivados de ácidos benzoicos sobre FSII e FSI e suas reações parciais}

Com o objetivo de determinar os sítios de interação na cadeia de transporte de electrons nos tilacóides, foram analisados os efeitos de 1 e $\mathbf{2}$ sobre o FSII desacoplado, FSI (Fotosistema I) e suas respectivas reações parciais, usando-se doadores e aceptores artificais de elétrons, bem como os inibidores apropriados. ${ }^{16}$ As substâncias $\mathbf{1}$ e 2 inibiram o fluxo de elétrons do FSII de $\mathrm{H}_{2} \mathrm{O}$ a DCBQ (2,5-dicloro-1,4-benzoquinona) (Figura 4), de $\mathrm{H}_{2} \mathrm{O}$ a SiMo (Figura 4) e, ainda, de DPC (difenilcarbazida) a DCPIP (2,6-diclofenol-indofenol) (Tabela 1). Os ácidos 1 e 2 apresentaram $\mathrm{I}_{50}$ de 113,0 e 138,0 $\mu \mathrm{M}$, respectivamente, para a reação FSII: $\mathrm{H}_{2} \mathrm{O}-$ DCBQ. Já para a reação parcial FSII: $\mathrm{H}_{2} \mathrm{O}$ - SiMo os valores de $\mathrm{I}_{50}$ obtidos foram de 151,0 (1) e 167,0 (2) $\mu \mathrm{M}$. Verificou-se ainda que 1 e 2 inibiram em 46 e $48 \%$, respectivamente, a $300 \mu \mathrm{M}$ a reação FSII: DPC - DCPIP (Tabela 1).

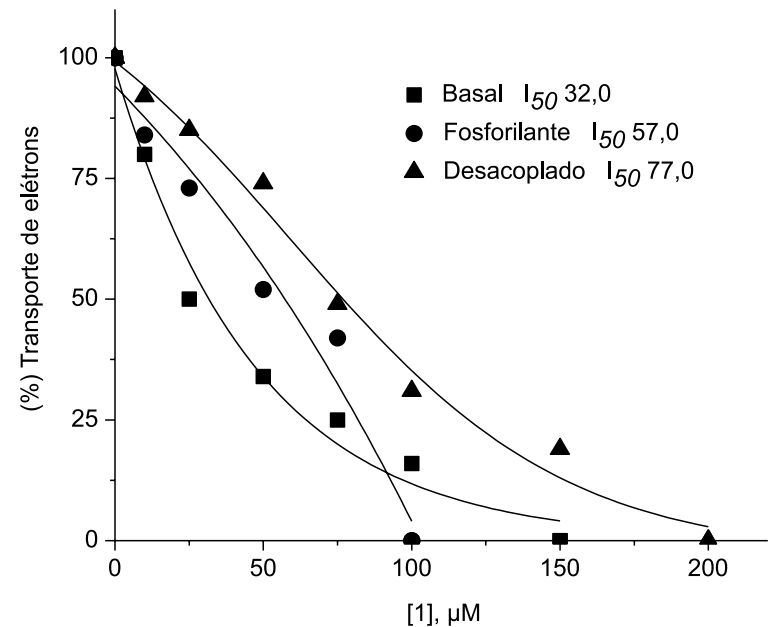

Figura 2. Efeito do composto 1 sobre o fluxo de elétrons (basal, fosforilante e desacoplado) de $\mathrm{H}_{2} \mathrm{O}-\mathrm{MV}$ em cloroplastos isolados das folhas de espinafre.

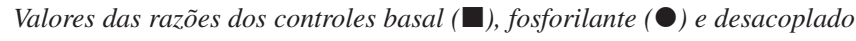

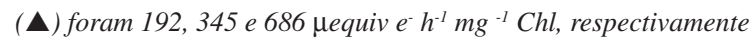

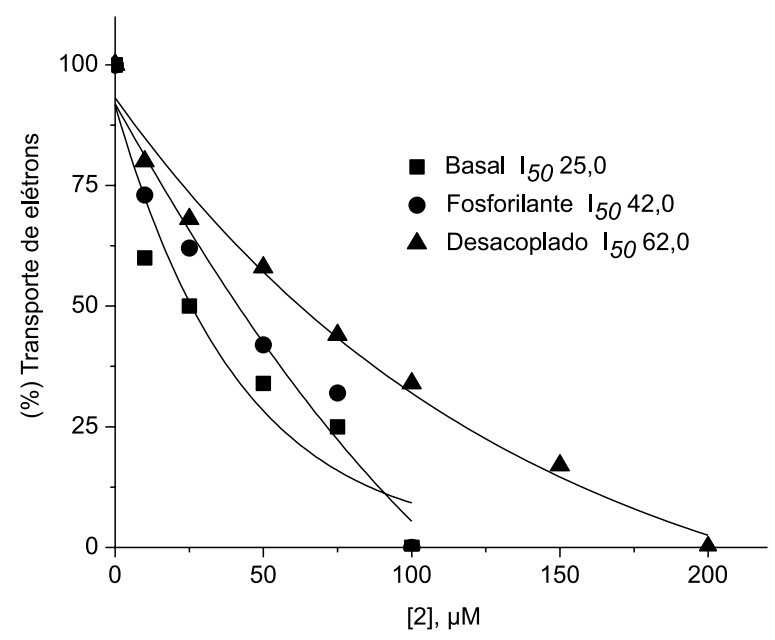

Figura 3. Efeito do composto 2 sobre o fluxo de elétrons (basal, fosforilante e desacoplado) de $\mathrm{H}_{2} \mathrm{O}-\mathrm{MV}$ em cloroplastos isolados das folhas de espinafre.

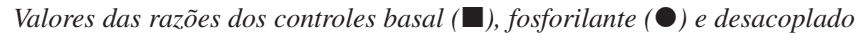
(வ) foram 192, 345 e $686 \mu$ equiv e- $h^{-1} \mathrm{mg}^{-1}$ Chl, respectivamente

As medidas polarográficas indicaram que o transporte de elétrons no FSII é afetado na presença dos compostos 1 e 2, prendendo-se ao sítio da ação da quinona $A\left(Q_{A}\right)$, impedindo a transferência de elétrons entre o centro reacional do FSII $\left(\mathrm{P}_{680}\right)$ e $\mathrm{Q}_{\mathrm{A}}$. Pode-se dizer então que as substâncias $\mathbf{1}$ e $\mathbf{2}$ afetam o FSII tanto pelo lado doador quanto pelo lado aceptor de elétrons, prendendo-se ao sítio catalítico localizado entre $\mathrm{P}_{680}-\mathrm{Q}_{\mathrm{A}}$ (pool das quinonas). A criação de centros de reação "silenciosos" pode ser um dos mecanismos de ação de $\mathbf{1}$ e $\mathbf{2}$ e ainda representa um caminho útil para o desenvolvimento de modelos para herbicidas inibidores da fotossíntese de plantas daninhas. Por outro lado, esses compostos não apresentaram efeito sobre o FSI (dados não apresentados).

\section{Medidas dos transientes da fluorescência da $\mathrm{Chl}$ a na presença dos compostos 1 e 2}

No intuito de colaborar para o esclarecimento do(s) sítio(s) de interação das substâncias $\mathbf{1}$ e $\mathbf{2}$ sobre o FSII, cloroplatos frescos e lisos foram incubados por 10 min no escuro, à temperatura ambiente com 


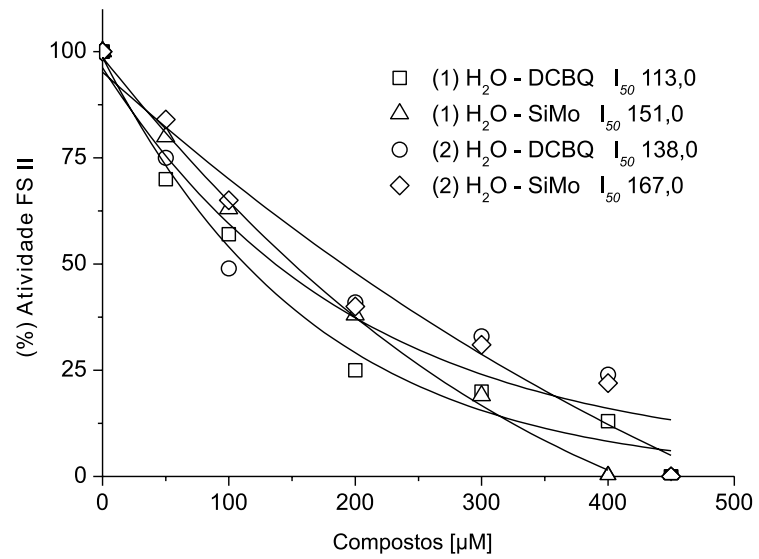

Figura 4. Efeito dos compostos 1 e 2 sobre o fluxo de elétrons no FSII: $\mathrm{H}_{2} \mathrm{O}-\mathrm{DCBQ}$ e FSII: $\mathrm{H}_{2} \mathrm{O}$ - SiMo em cloroplastos isolados das folhas de espinafre. $O$ valor da razão do controle foi de 545 e $356 \mu$ equiv $e^{-} h^{-1} \mathrm{mg}^{-1}$ Chl, respectivamente

Tabela 1. Efeito dos compostos 1 e $\mathbf{2}$ sobre o transporte de elétrons do FSII desacoplado de DPC - DCPIP

\begin{tabular}{|c|c|c|c|c|}
\hline & $\begin{array}{l}\text { mols DCPIP }{ }_{\text {red }} / \\
\mathrm{mg} \mathrm{Chl} \mathrm{h}^{\text {m }}\end{array}$ & $\%$ & $\begin{array}{c}\text { mols DCPIP }_{\text {red }} / \\
\mathrm{mg} \mathrm{Chl} \mathrm{h}\end{array}$ & $\%$ \\
\hline [ ] & 1 & & 2 & \\
\hline 0 & 240 & 100 & 240 & 100 \\
\hline 50 & 202 & 84 & 228 & 95 \\
\hline 100 & 173 & 72 & 184 & 76 \\
\hline 200 & 170 & 70 & 150 & 62 \\
\hline 300 & 130 & 54 & 125 & 52 \\
\hline
\end{tabular}

$200 \mu \mathrm{M}$ de cada uma das substâncias, DCMU $50 \mu \mathrm{M}$ e Tris $0,8 \mathrm{M}$, os quais foram usados como controle positivo (Figura 5). O controle dos tilacoides mostrou uma curva de fluorescência polifásica com uma sequência OJIP de transientes similares àqueles previamente publicados por Strasser et al..${ }^{17} \mathrm{~A}$ adição de $50 \mu \mathrm{M}$ do herbicida DCMU induz um rápido aumento do rendimento da fluorescência durante os primeiros $2 \mathrm{~ms}$ de iluminação; isto transforma a sequência regular OJIP numa curva OJ. ${ }^{17}$ Quando os cloroplastos são tratados com tris,

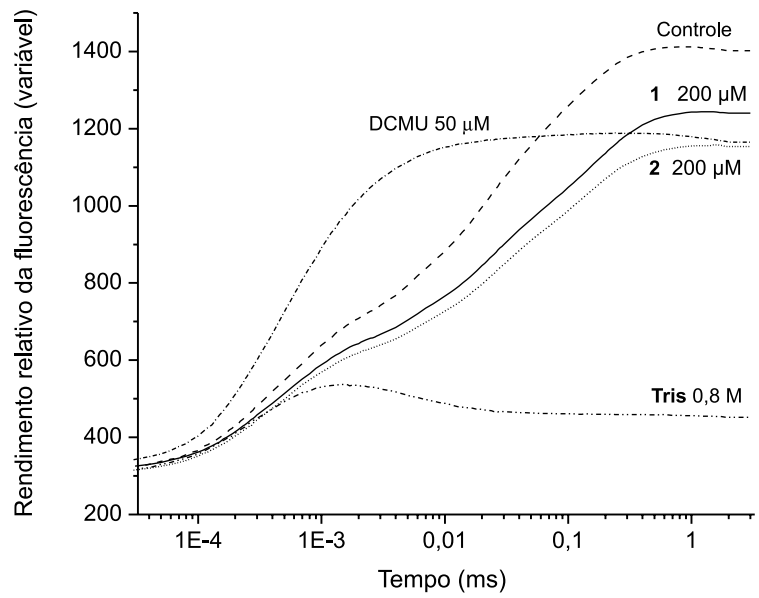

Figura 5. Aumento cinético da fluorescência de cloroplastos frescos e lisos infiltrados com os ácidos 1 e 2, DCMU $50 \mu \mathrm{M}$ e tilacoides tratados com Tris 0,8 M. Os controles dos cloroplastos são mostrados para comparação. As curvas de indução de fluorescência (Chl a) foram medidas à temperatura ambiente. Os dados são médias de cinco repetições verifica-se a redução na indução da fluorescência. ${ }^{18}$ Isto resulta na redução do rendimento máximo da fluorescência, a banda-K aparece e consiste de um rápido aumento (a $300 \mu$ s) seguido por uma redução no rendimento da fluorescência a um nível próximo a $F_{0}$ (fluorescência inicial onde todos os níveis, J e I estão ausentes do transiente). ${ }^{19,20} \mathrm{As}$ condições dos tilacoides tratados com tris são mostradas também por cloroplastos tratados-aquecidos onde as perdas no OEC (complexo de evolução de oxigênio) ocorrem.

A análise da diferença de cada curva do controle da fluorescência relativa variável, quando a fase $\mathrm{O}-\mathrm{J}$ foi normalizada entre $F_{\mathrm{j}}-F_{0}$, $W_{\mathrm{t}}=F_{\mathrm{v}, \mathrm{l}} /\left(F_{\mathrm{j}}-F_{0}\right) /\left(F_{\mathrm{j}}-F_{0}\right)$, mostra um aumento na supressão do nível $\mathrm{J}$ (a $2 \mathrm{~ms}$ ) e um rápido aumento a aproximadamente $300 \mu \mathrm{s}$ aparece na presença de $\mathbf{1}$ e $\mathbf{2}$, indicando claramente a criação de uma banda K (Figura 6). O aparecimento da banda K surge a partir de um desequilíbrio no fluxo de elétrons entre os doadores e aceptores de elétrons do sítio do FSII. Nesta condição, o fluxo de elétrons do OEC a $\mathrm{Z}$ (resíduo de tirosina) é mais lento que o fluxo de elétrons do $\mathrm{P}_{680}$ $\mathrm{a} \mathrm{Q}_{\mathrm{A}}$. $\mathrm{O}$ rápido aumento da fluorescência inicial é devido à redução de $\mathrm{Q}_{\mathrm{A}}$ a $\mathrm{Q}_{\mathrm{A}}{ }^{-}$seguida pela redução de $\mathrm{P} 680^{+}$por $\mathrm{Z}$ sem a participação direta do complexo OEC. ${ }^{19}$

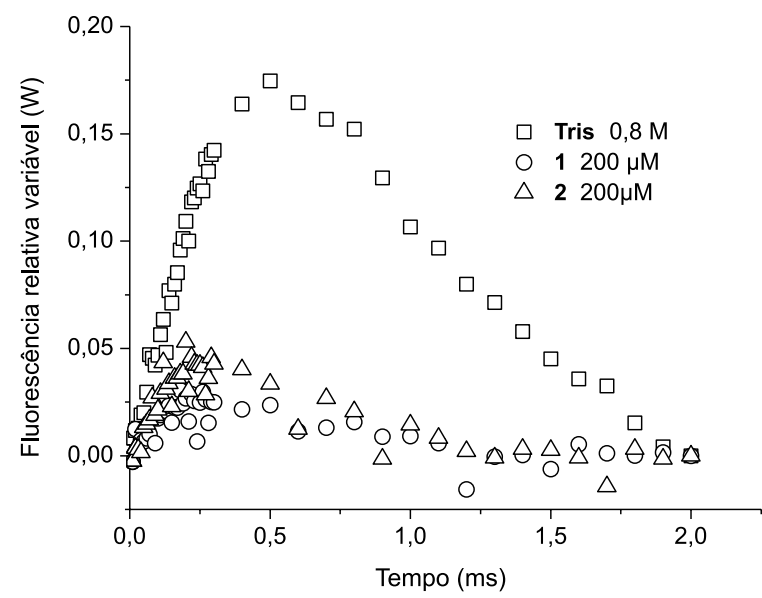

Figura 6. Aparecimento da banda Kem aproximadamente $300 \mu$ s. Diferença de cada curva do controle com a fluorescência variável relativa normalizada na amplitude $F_{j}-F_{0}$. Ácido 192 a $200 \mu M(\mathrm{o})$ e derivado 193 a $200 \mu \mathrm{M}(\triangle)$. Cloroplastos incubados com 0,8 M Tris ( $\square$ )

A análise do transiente da fluorescência ( $\mathrm{Chl} a$ ) claramente indica que o fluxo de elétrons no OEC foi inibido pelos compostos. Quando o OEC é inibido, não há doação de elétrons a $Y_{z}$, que por sua vez não reduz $\mathrm{P}_{680}{ }^{+}$; dentro deste contexto, ocorre uma máxima acumulação de $\mathrm{P}_{680}{ }^{+}$, $\mathrm{Pheo}^{+}$e $\mathrm{Q}_{\mathrm{A}}$. Strasset et al. ${ }^{21}$ propuseram o termo "centro de reação silencioso" como os centros que não podem nem reduzir $\mathrm{Q}_{\mathrm{A}} \mathrm{e}$ nem voltar a transferir sua energia de excitação às moléculas antena (clorofilas); portanto, as unidades correspondentes ao FSII não contribuem para a fluorescência variável e seus rendimentos de fluorescência são menores. ${ }^{20}$ Os valores de $F_{0}$ foram quase constantes e de $F_{\mathrm{M}}$ diminuíram na presença de $\mathbf{1}$ e $\mathbf{2}$, e os valores mais baixos da área sobre a curva entre $F_{0}$ e $F_{\mathrm{M}}$ (Tabela 2) indicam que a transferência de elétrons ao sítio da quinona é bloqueada. Assim, a quinona $A\left(Q_{A}\right)$ do FSII não é reduzida, e estes resultados sugerem a criação dos centros de reação silenciosos, ${ }^{20}$ devido à interferência das substâncias $\mathbf{1}$ e $\mathbf{2}$.

\section{PARTE EXPERIMENTAL}

\section{Procedimentos gerais}

Os experimentos de RMN 1D $\left({ }^{1} \mathrm{H},{ }^{13} \mathrm{C}\right)$ foram adquiridos em 
Tabela 2. Efeito do DCMU, das substâncias 1 e 2 sobre os parâmetros da fluorescência de tilacoides previamente incubados por $10 \mathrm{~min}$ no escuro, e com tris $0,8 \mathrm{M}, \mathrm{pH} 8,0$

\begin{tabular}{lcccc}
\hline Substância & $\mathrm{F}_{0}$ & $\mathrm{~F}_{M}$ & $\mathrm{~F}_{V} / \mathrm{F}_{M}$ & Área \\
\hline Controle & 304 & 1412 & 0,785 & 48821 \\
DCMU $(50 \mu \mathrm{M})$ & 305 & 1188 & 0,743 & 2421 \\
Tris $(0,8 \mathrm{M})$ & 299 & 537 & 0,443 & 0 \\
$\mathbf{1}(200 \mu \mathrm{M})$ & 308 & 1244 & 0,752 & 65648 \\
$\mathbf{2}(200 \mu \mathrm{M})$ & 297 & 1158 & 0,744 & 58925 \\
\hline
\end{tabular}

$\mathrm{CDCl}_{3}$ em espectrômetro Bruker Avance III - 9,4 Tesla (400 MHz para frequência do hidrogênio e $100 \mathrm{MHz}$ para carbono), equipado com trocador automático de amostra e sonda de $5 \mathrm{~mm}$ BFO (smart probe com $\left.\mathrm{ATMA}^{\circledR}\right)$.

\section{Material vegetal}

Os frutos de Myrsine cuneifolia foram coletados no mês de setembro de 2008, em Anápolis-Goiás, no Campus da Universidade Estadual de Goiás, e identificados pela $\operatorname{Prof}^{a} \operatorname{Dr}^{\mathrm{a}}$ M. L. dos Santos. A exsicata, $n^{\circ} .4215$, do material vegetal foi depositada no herbário do departamento de Ciências Biológicas da Universidade Estadual de Goiás.

\section{Extração e isolamento dos constituintes}

Os frutos secos de M. cuneifolia (400 g) foram macerados e extraídos exaustivamente em etanol a frio. O solvente foi evaporado à pressão reduzida em evaporador rotatório $\left(45^{\circ} \mathrm{C}\right)$, o que permitiu a obtenção de $62 \mathrm{~g}$ do extrato bruto etanólico (MCFrE). Esse extrato foi fracionado por cromatografia em coluna $(\phi=3,0 \mathrm{~cm} \mathrm{x} \mathrm{h}=20,0$ $\mathrm{cm}$ ), utilizando-se como fase estacionária celulose microcristalina D e eluentes em ordem crescente de polaridade: hexano, diclorometano, acetato de etila e metanol, obtendo-se as frações: hexânica (MCFrEH - $10 \mathrm{~g}$ ), diclorometânica (MCFrED - $4 \mathrm{~g}$ ), acetato de etila (MCFrEA - $5 \mathrm{~g}$ ) e metanólica (MCFrEW - $30 \mathrm{~g}$ ).

A fração MCFrEH (1 g) foi fracionada por cromatografia em coluna utilizando como fase estacionária sílica flash (230-400 Mesh) e como fase móvel diclorometano-acetato de etila $5 \%(\phi=3,0 \mathrm{~cm} \mathrm{x}$ $\mathrm{h}=19,0 \mathrm{~cm}$ ), o que gerou 22 frações. A reunião das frações $5-12$, forneceu a o ácido myrsinoico $1(0,4 \mathrm{~g})$.

Ácido 3-geranil-4-hidroxi-5-(3"-metil-2”-butenil)-benzóico: Ácido myrsinoico A (1)

Óleo amarelado. RMN de ${ }^{1} \mathrm{H}\left[400 \mathrm{MHz}, \mathrm{CDCl}_{3}, \delta, J(\mathrm{~Hz})\right]: 7,68$ $(s ; \mathrm{H}-2), 7,68$ ( $s$; H-6), 3,33 ( $\left.d ; J=7,2 ; \mathrm{H}^{-1}{ }^{\prime}\right), 5,01\left(t l ; J=7,2 ; \mathrm{H}-2^{\prime}\right)$, 2,03 ( $s$; H-4'), 2,03 ( $s$; H-5'), 5,01 ( $t l ; J=7,2 ; \mathrm{H}-6$ '), 1,61 ( $s ; \mathrm{H}-8^{\prime}$ '), 1,53 ( $s$; H-9'), 1,70 ( $s$; H-10'), 3,33 ( $s$; H-1"), 5,25 ( $t l ; J=7,2$; H-2”). RMN de ${ }^{13} \mathrm{C}\left[100 \mathrm{MHz}, \mathrm{CDCl}_{3}, \delta\right]: 130,0$ (C-1); 131,6 (C-2); 121,0 (C-3); 157,4 (C-4); 123,5 (C-5); 127,0 (C-6); 29,0 (C-1'); 120,8 (C2'); 138,6 (C-3'); 39,4 (C-4'); 26,0 (C-5'); 126,6 (C-6'); 134,5 (C-7'); 25,3 (C-8'); 17,3 (C-9'); 15,9 (C-10'); 29,3 (C-1”); 120,9 (C-2”); 132,6 (C-3"); 25,4 (C-4”); 17,5 (C-5”); 170,4 (COOH).

\section{Hidrogenação catalítica de 1}

Solubilizou-se 0,02 g da substância (1) em $4 \mathrm{~mL}$ de metanol, seguida da adição de $0,01 \mathrm{mg}$ de paládio adsorvido em carvão $(\mathrm{Pd} / \mathrm{C})$. O sistema permaneceu sob atmosfera de gás hidrogênio durante 12 h, sob agitação constante; após este tempo filtrou-se sob celite, o que permitiu a obtenção do derivado (2) $(0,017 \mathrm{~g})$.
Derivado hidrogenado (2)

Sólido branco amorfo. RMN de ${ }^{1} \mathrm{H}\left[400 \mathrm{MHz}, \mathrm{CDCl}_{3}, \delta, J(\mathrm{~Hz})\right]$ : 7,68 ( $s ; \mathrm{H}-2), 7,68$ ( $s ; \mathrm{H}-6), 0,87\left(d ; J=6,6 ; 2 \mathrm{CH}_{3}\right), 0,97(d ; J=6,6$; $\left.2 \mathrm{CH}_{3}\right), 0,96\left(d ; J=6,6 ; \mathrm{CH}_{3}\right), 2,62(m ; \mathrm{H}-1 '), 2,62\left(m ; \mathrm{H}-1{ }^{\prime \prime}\right), 1.11-$ 1,67 (m, H-1', H-2', H-3', H-4', H-5', H-6', H-7', H-2'” e H-3')).

\section{Avaliação do potencial herbicida: inibição da reação de Hill}

As folhas de espinafre foram obtidas nos supermercados da cidade de São Carlos - SP, em seguida lavadas e secas, para posterior seleção das folhas com maior grau de uniformidade. Para extração dos cloroplastos de espinafre ( $S$. oleracea L.), as folhas já livres da nervura central e das extremidades basal e apical (25 g) foram trituradas em liquidificador e submetidas à extração com $100 \mathrm{~mL}$ do seguinte meio: sacarose $\left(400 \mathrm{mmol} \mathrm{L}^{-1}\right), \mathrm{MgCl}_{2}\left(5 \mathrm{mmol} \mathrm{L}^{-1}\right), \mathrm{KCl}$ (10 $\left.\mathrm{mmol} \mathrm{L}^{-1}\right)$ e $\mathrm{K}^{+}$-tricina $\left(30 \mathrm{mmol} \mathrm{L}^{-1}\right)$ em pH 8,0 (1 mol L-1 $\left.\mathrm{KOH}\right)$. $\mathrm{O}$ meio foi homogeneizado e filtrado com o auxílio de um funil e camadas de gaze. O filtrado foi centrifugado ( $4000 \mathrm{rpm}, 4^{\circ} \mathrm{C}$ e $5 \mathrm{~min}$ ) e o sobrenadante, eliminado. O precipitado contendo os cloroplastos foi dissolvido em $1 \mathrm{~mL}$ do mesmo meio usado no isolamento. ${ }^{22}$

Para determinar a concentração de clorofila, duas alíquotas de 20 $\mu \mathrm{L}$ da suspensão de cloroplastos foram transferidas para dois tubos de centrífuga contendo $5 \mathrm{~mL}$ de acetona $(80 \%)$, cada. A amostra permaneceu no escuro por 5 min e, depois, foi submetida à centrifugação (4000 rpm e 5 min). Em seguida, foram feitas medidas de absorbâncias do sobrenadante nos comprimentos de onda de 645 e $663 \mathrm{~nm}$, de acordo com a Equação $1 .{ }^{23}$

$$
\text { Clorofila }\left(\mu \mathrm{g} \mathrm{mL}^{-1}\right)=20,2\left(\mathrm{~A}_{645}\right)+8,02\left(\mathrm{~A}_{663}\right)
$$

Para os ensaios foram utilizadas soluções dos compostos puros, caracterizados e identificados. As soluções das amostras foram preparadas em concentrações variadas, usando solução DMSO (0,5\%) como solvente. $\mathrm{O}$ fluxo de elétrons fotossintético foi determinado pela captura de oxigênio, utilizando-se um microeletrodo de Clark conectado ao medidor de oxigênio dissolvido, YSI 5300A. Para avaliar a ação das amostras na inibição da reação de Hill, estas foram adicionadas ao meio reacional em concentrações que variaram de 1 a $500 \mu \mathrm{mol} \mathrm{L}{ }^{-1}$ e as medidas foram comparadas com o controle. ${ }^{24}$ Para o transporte de elétrons basal, utilizou-se o seguinte meio reacional: clorofila $\left(20 \mu \mathrm{g} \mathrm{mL}^{-1}\right)$, sacarose $(100 \mathrm{mmol} \mathrm{L}-1), \mathrm{MgCl}_{2} \cdot 6 \mathrm{H}_{2} \mathrm{O}\left(5 \mathrm{mmol} \mathrm{L}{ }^{-1}\right)$, $\mathrm{KCl}\left(10 \mathrm{mmol} \mathrm{L}^{-1}\right)$ e $\mathrm{K}_{3}\left[\mathrm{Fe}(\mathrm{CN})_{6}\right]\left(\mathrm{K}^{+}\right.$-tricina $), 15 \mathrm{mmol} \mathrm{L}^{-1}, \mathrm{pH} 8$ (1 mmol L-1 $\mathrm{KOH})$. A reação teve início com iluminação saturante de $350 \mathrm{~W}$ (lâmpada comum) durante 3 min, sob condições aeróbicas. ${ }^{25}$ Utilizou-se $100 \mu \mathrm{M}$ de MV [dicloreto de 1,1'-dimetil-4,4'-bipiridínio (dicloreto de paraquat)] como aceptor de elétrons. O transporte de elétrons fosforilante foi determinado, assim como o transporte de elétrons basal, adicionando-se à mistura uma solução $1 \mathrm{mmol} \mathrm{L}^{-1} \mathrm{de}$ ADP e $3 \mathrm{mmol} \mathrm{L}^{-1} \mathrm{~K}_{2} \mathrm{HPO}_{4}, \mathrm{KCN}\left(0,1 \mathrm{mmol} \mathrm{L}^{-1}\right)$ para inibir a atividade catalítica. $\mathrm{O}$ transporte de elétrons desacoplado foi determinado da mesma forma que o transporte de elétrons basal, adicionando-se à mistura uma solução $5 \mathrm{mmol} \mathrm{L}^{-1}$ de $\mathrm{NH}_{4} \mathrm{Cl}$. As reações parciais na cadeia de transporte de elétrons foram medidas utilizando-se inibidores específicos, como DCMU $10 \mu \mathrm{mol} \mathrm{L} \mathrm{L}^{-1}$; 2,5-dibromo-3-metil-6-isopropil-1,4-p-benzoquinona (DBMIB) $1 \mu \mathrm{mol} \mathrm{L} \mathrm{L}^{-1}$; e os seguintes doadores e aceptores de elétrons: silicomolibdato de sódio $\left(\mathrm{SiMo} \mathrm{Na}^{+}\right)$ $100 \mu \mathrm{mol} \mathrm{L}{ }^{-1}$; difenilcarbazida (DPC) $200 \mu \mathrm{mol} \mathrm{L}^{-1}$; diaminodureno (DAD), $100 \mu \mathrm{mol} \mathrm{L}^{-1}$ e metilviologênio (MV) $100 \mu \mathrm{mol} \mathrm{L}^{-1}$.

\section{Determinação do fluxo de elétrons nos fotossistemas II e I (FSII} e FSI) desacoplados e suas reações parciais

O fluxo de elétrons nos FSII e FSI foi medido, assim como no 
ensaio para o transporte de elétrons desacoplado. O FSII inicialmente foi medido de $\mathrm{H}_{2} \mathrm{O}$ a DCPIP reduzido, ou $\mathrm{H}_{2} \mathrm{O}$ a DCBQ, suportados pela evolução de $\mathrm{O}_{2}$. O meio reacional foi constituído por $1 \mu \mathrm{M}$ DBMIB, $100 \mu \mathrm{M}$ DCPIP, $500 \mu \mathrm{M} \mathrm{K}_{3}\left[\mathrm{Fe}(\mathrm{CN})_{6}\right]$ e $6 \mathrm{mM} \mathrm{NH}_{4} \mathrm{Cl}^{25}$ Em seguida, a substância foi submetida ao transporte de elétrons do FSII $\left(\mathrm{H}_{2} \mathrm{O}\right.$ a SiMo), que foi determinado assim como para FSII, com exceção de $1 \mu \mathrm{M}$ DBMIB, $100 \mu \mathrm{M}$ DCPIP, $500 \mu \mathrm{M} \mathrm{K}_{3}\left[\mathrm{Fe}(\mathrm{CN})_{6}\right]$, que foram omitidos e $200 \mu \mathrm{M}$ SiMo e $10 \mu \mathrm{M}$ DCMU, que foram adicionados. ${ }^{26}$

O transporte de elétrons do FSII (DPC a DCPIP ${ }_{\text {oxidado }}$ ) foi medido através da redução da razão de DCPIP $_{\text {ox }}$ com espectrofotômetro e determinado em tilacoides previamente tratados com Tris $0,8 \mathrm{M}$ ( $\mathrm{pH} 8,0$ ) e incubados por $30 \mathrm{~min}$ a $4{ }^{\circ} \mathrm{C} .{ }^{27}$ Quando um composto inibe as reações do fotossistema II, o sítio de inibição é comprovado utilizando-se a técnica de fluorescência transiente da clorofila $a$ do FSII. A razão de transporte de elétrons do FSI foi determinada através do consumo de $\mathrm{O}_{2}$ de uma maneira similar à razão do transporte de elétrons basal não cíclico com $10 \mu \mathrm{M}$ DCMU, $100 \mu \mathrm{M}$ DCPIP reduzido com $300 \mu \mathrm{M}$ ácido ascórbico, $50 \mu \mathrm{M} \mathrm{MV}$ e $6 \mathrm{mM} \mathrm{NH}_{4} \mathrm{Cl}$, que foram utilizados como meio reacional. ${ }^{28}$

\section{Estudos da fluorescência da clorofila $a(\mathrm{Chl} a)$}

As curvas de indução da Chl $a$ foram medidas à temperatura ambiente com um Hansatech Handy PEA (Plant Effiecient Analyzer) como descrito por Strasser et al.. ${ }^{17} \mathrm{O}$ rendimento máximo da fluorescência das amostras foi gerado usando três diodos que emitem luz $(650 \mathrm{~nm})$. A duração do pulso foi de $1 \mathrm{~s}$. O meio de reação utilizado foi o mesmo que para as medidas do transporte de elétrons basal. Para monitorar os transientes da fluorescência da Chl $a$, alíquotas de tilacoides adaptados ao escuro contendo $20 \mu \mathrm{g}$ de Chl foram transferidas a um papel de filtro $(1,0 \times 1,0 \mathrm{~cm})$. Em seguida, o material foi adicionado em $3 \mathrm{~mL}$ do meio e ainda em diferentes concentrações dos compostos testados. As amostras foram mantidas no escuro por 10 min e, em seguida, foram realizadas as leituras dos parâmetros. Os resultados representam a média de cinco repetições, obtidas diretamente através do software PEA Plus Versão 1.02.

Os diferentes parâmetros técnicos da fluorescência do FSII foram obtidos com a equação do teste OJIP, usando o programa Bioanalyser.$^{29} \mathrm{~A}$ área entre a curva de fluorescência máxima $\left(\mathrm{F}_{\mathrm{M}}\right) \mathrm{e}$ inicial $\left(\mathrm{F}_{0}\right)$ foi alterada pela presença de compostos que interagem com o FSII. ${ }^{17,20}$ A energia medida precisa bloquear todos os centros de reação do FSII, que foi medida pela razão Área/(Fm - Fo). $V_{J}$, fluorescência da eficiência quântica variável relativa a $2 \mathrm{~ms}$ (fase $\mathrm{J}$ ). A medida de $\mathrm{V}_{\mathrm{J}}=(\mathrm{F} 2 \mathrm{~ms}-\mathrm{Fo}) /\left(\mathrm{F}_{\mathrm{M}}-\mathrm{F}_{0}\right)$. $\mathrm{V}_{\mathrm{J}}$ é usada como indicador da eficiência fotoquímica primária do FSII. $\mathrm{V}_{\mathrm{I}}$ representa a fluorescência variável relativa a $30 \mathrm{~ms}$. A medida de $\mathrm{V}_{\mathrm{I}}=\left(\mathrm{F} 30 \mathrm{~ms}-\mathrm{F}_{0}\right) /\left(\mathrm{F}_{\mathrm{M}}-\mathrm{F}_{0}\right)$; Sm, é a área normalizada. ${ }^{29}$

A densidade dos centros de reação, $\mathrm{RC} / \mathrm{Cs}$, fornece o número ativo proporcional aos centros reacionais $(\mathrm{RCs})$ para a seção cruzada da amostra medida, RC/CSo $=\mathrm{PHI}\left(\mathrm{P}_{0}\right)\left(\mathrm{V}_{\mathrm{J}} / \mathrm{M}_{0}\right) \mathrm{F}_{0}$; índice de desempenho, $\mathrm{PI}(\mathrm{abs})=(\mathrm{RC} / \mathrm{ABS})\left[\mathrm{PHI}\left(\mathrm{P}_{0}\right) /\left(1-\mathrm{PHI}\left(\mathrm{P}_{0}\right)\right]\left[\mathrm{PSI}_{0} /\left(1-\mathrm{PSI}_{0}\right)\right]\right.$. $\mathrm{PSI}_{0}$ expressa a probabilidade de excitação aprisionada pela entrada no centro de reação do FSII da cadeia do transporte de elétrons, PSIo $=\operatorname{Area} /\left(\mathrm{F}_{\mathrm{M}}-\mathrm{F}_{0}\right) \mathrm{V}_{\mathrm{J}}$

$\mathrm{O}$ número de turnover de $\mathrm{Q}_{\mathrm{A}}$ indica quantas vezes $\mathrm{Q}_{\mathrm{A}}$ é reduzida no tempo $\mathrm{t}_{0} \mathrm{a}_{\mathrm{Fmax}}$ e é medida por $\mathrm{N}=\mathrm{Sm} . \mathrm{Mo}(1 / \mathrm{VJ})$; $\mathrm{Sm}$ é a área complementar total normalizada, que corresponde à fase $\mathrm{O}-\mathrm{J}_{\mathbf{0}} \mathrm{M}_{0}$ fornece a inclinação da curva de normalização desde a origem até o aumento da fluorescência, bem como a medida da taxa fotoquímica primária. ${ }^{30}$ Calcula-se $\mathrm{M}_{0}=4\left(\mathrm{~F} 300-\mathrm{F}_{0}\right) /\left(\mathrm{F}_{\mathrm{M}}-\mathrm{F}_{0}\right)$ por $1 \mathrm{~ms}$.

PHI(Po) ou TRo/ABS (eficiência quântica ou relações de fluxo) representam a probabilidade de captura de elétrons ou rendimento da eficiência quântica, expressa a probabilidade de um fóton ser absorvido e preso pelo centro da reação PSII. Calcula-se $\mathrm{PHI}(\mathrm{Po})=(\mathrm{Fm}($ Área $) /(\mathrm{FM}-\mathrm{Fo}) \mathrm{Fo}) / \mathrm{Fm}$. A constante de excitação fotoquímica $\left(\mathrm{k}_{\mathrm{P}}\right)$ é dada por $\mathrm{k}_{\mathrm{p}}=(\mathrm{ABS} / \mathrm{CS}) \mathrm{k}_{\mathrm{F}}[(1 / \mathrm{Fo}) \mathrm{Area} /(\mathrm{Fm}-\mathrm{Fo})$ $(1 / \mathrm{Fm})]$. Tem-se ainda $\mathrm{k}_{\mathrm{N}}$, constante de excitação não fotoquímica; $\mathrm{K}_{\mathrm{H}}$ (constante por dissipação de calor) e $\mathrm{K}_{\mathrm{F}}$ (emissão por fluorescência). Calcula-se $\mathrm{k}_{\mathrm{N}}=(\mathrm{ABS} / \mathrm{CS}) \mathrm{k}_{\mathrm{F}}(1 / \mathrm{Fm})$. ABS/RC fornece o tamanho efetivo da antena do centro de reação ativo (RC). Este parâmetro expressa o número total de fótons absorvidos pela molécula de clorofila em todos os centros reacionais, $\mathrm{ABS} / \mathrm{RC}=\mathrm{M}_{0}\left(1 / \mathrm{V}_{\mathrm{J}}\right)\left(1 / \mathrm{PHI}\left(\mathrm{P}_{0}\right)\right.$.

\section{CONCLUSÕES}

A partir dos frutos de Myrsine cuneifolia foi isolado o composto 1 (ácido myrsinoico A). Esse metabólito sofreu reação de hidrogenação catalítica, para a obtenção de um derivado. Neste trabalho, relata-se pela primeira vez o efeito desses compostos sobre a fotossíntese in vitro em cloroplastos de espinafre, através de uma série de reações enzimáticas, utilizadas para o esclarecimento de seus mecanismos de ação. Os resultados sugeriram que os compostos possuem mais de um mecanismo de ação. Os compostos que inibem o transporte de elétrons em todas as suas etapas (basal, fosforilante e desacoplado) atuam como inibidores da reação de Hill, através do bloqueio e/ou redução do transporte de elétrons pelo lado aceptor de elétrons (neste caso, o MV). Na literatura são encontrados outros produtos naturais que se comportam de maneira similar: ácido trachiloban-19-oico, ${ }^{31}$ xantorrizol, ${ }^{25}$ tricolorin $\mathrm{A},{ }^{24} \mathrm{~N}$-metil-flindersina, ${ }^{11}$ 6-(3,3-dimetilaliloxi)-4-metoxi-5-metilfitalideo, ${ }^{32}$ butil $7 \beta$-hidroxi-6-oxovouacapan-17 $\beta$-oate, ${ }^{33}$ canofilol ${ }^{34}$ e 1,2,3,4,5,6-hexa- $O$-acetildulcitol. ${ }^{35}$ Dados obtidos após medidas polarográficas permitiram localizar o sítio de ação dos compostos sobre o FS II. Ambos possuem sítio de interação tanto pelo lado doador, quanto pelo aceptor de elétrons, prendendo-se ao pool das quinonas $\left(P_{680}-\mathrm{Q}_{\mathrm{A}}\right)$. Um terceiro mecanismo de ação foi descoberto através de estudos de fluorescência da clorofila $a(\mathrm{ChL} a)$, que indicaram que os metabólitos inibem parcialmente o complexo enzimático que provoca a fotólise da molécula de água, ou seja, ocorre a formação de centros de "reação silenciosos", o que não permite a redução do aceptor primário de elétrons do FS II $\left(\mathrm{Q}_{\mathrm{A}}\right)$, impedindo a transferência de elétrons. Devido à quantidade razoável obtida de ácido myrsinoico $1(0,4 \mathrm{~g})$, posteriormente esse composto passará por novas modificações estruturais, no intuito de se obter derivados mais ativos e com maior potencial fitotóxico.

\section{AGRADECIMENTOS}

Ao CNPq, à CAPES e FAPESP pelo suporte financeiro.

\section{REFERÊNCIAS}

1. Le Bourlegat, C. A. Em Fragmentação florestal e alternativas de desenvolvimento rural na região centro-oeste; Costa, R. B., ed.; Universidade Católica Dom Bosco: Campo Grande, 2003.

2. Borlaug, N. E. Em Feeding a world of 10 billion people: the miracle ahead. Global warming and other eco-myths; Bailey R., ed.; Competitive Enterprise Institute: Roseville, 2002.

3. Reguero, M. T.; Calle, J.; Mata, R.; Rev. Colomb. Ciencias Quim. Farm. 1989, 17, 57.

4. Cordero, C. P.; Gómez-González, S.; León-Acosta, C. J.; MorantesMedina, S. J.; Aristizabal, F. A.; Fitoterapia 2004, 75, 225.

5. Kimura, Y.; Misuno, T.; Shimada, A.; Tetrahedron 1997, 38, 469.

6. Kimura, Y.; Misuno, T.; Shimada, A.; Kawano, T.; Phytochemistry 1998, 47, 323.

7. Kimura, Y.; Shimada, A.; Kusano, M.; Yoshii, K.; Morita, A.; Nishibe, M.; Fujioka, S.; Kawano, T.; J. Nat. Prod. 2002, 65, 621. 
8. Pillmoor, J. B.; Pestic. Sci. 1998, 52, 75.

9. Greaves, M. P. Em Crop Protection Agents from Nature: Natural Products and Analogues; Copping, L. G., ed.; RCS: Cambridge, 1996.

10. Duke, S. O.; Romagni, J. G.; Dayan, F. E.; Crop Protection 2000, 19, 583.

11. Veiga, T. A. M.; González-Vázquez, R.; Braga, P. A. C.; da Silva, M. F. G. F.; King-Díaz, B.; Lotina-Hennsen, B.; Allelopathy J. 2008, 21, 133.

12. Veiga, T. A. M.; Silva, S. C.; Archundia-Camacho, F.; Vieira, P. C.; Fernandes, J. B.; Rodrigues Fo, E.; Muller, M. W.; da Silva, M. F. das G. F.; Hensenn, B. L.; J. Agric. Food Chem. 2007, 55, 4217.

13. Veiga, T. A. M.; Gonzalez-Vazquez, R.; Oiano Neto, J.; da Silva, M. F. das G. F.; Diaz, B. K.; Hensenn, B. L.; Arc. Biochem. Biophys. 2007, 465,38 .

14. Mills, J. D.; Mitchell, P.; Schurmann, P.; FEBS Lett. 1980, 191, 144

15. Izawa, S.; Winget, C. D.; Good, N. E.; Biochem. Biophys. Res. Commun. 1972, 22, 222.

16. King-Díaz, B.; Macias-Ruvalcaba, N.; Aguilar-Martinez, M.; Calaminici, P.; Lotina-Hennsen, B.; J. Photochem. Photobiol. 2006, 83, 105.

17. Strasser, R. J.; Srivastava, A.; Govindjee; Photochem. Photobiol. 1995, $61,32$.

18. Rickert, K. W.; Sears, J.; Beck, W. F.; Brudvig, G. W.; Biochemistry 1991, 30, 7888 .

19. Strasser, R. J.; Photosynth. Res. 1997, 52, 147.

20. Strasser, R. J.; Tsimilli-Michael, M.; Srivastava, A.; Adv. Photos. Resp. 2004, 19, 321.

21. Lazár, D.; Pospísil, P.; Naus, J.; Photosynthetica 1999, 37, 255.

22. Macias, M. L.; Rojas, I. S.; Mata, R.; Lotina-Hennsen, B.; J. Agric. Food Chem. 1999, 47, 2137.
23. Romagni, J. G.; Nanayakkara, N. P. D.; Rosell, R.; Dayan, F. E. Em Allelopathy from Molecules to Ecosystems; Reigosa, M. J.; Pedrol, N., eds.; Enfield Science, Publishers: New Hampshire, 2002.

24. Achnine, L; Moreno-Sánchez, R.; Iglesias-Prieto, R.; Pereda-Miranda, R.; Lotina-Hennsen, B.; Physiol. Plant. 1999, 106, 246.

25. González-Bernardo, E.; King-Díaz, B.; Delgado, G.; Aguilar, M. A.; Lotina-Hennsen, B.; Physiol. Plant. 2003, 119, 598.

26. Giaquinta, R. T.; Selman, B. R.; Anderson, B. J.; Dilley, R. A.; J. Biol. Chem. 1974, 249, 2873.

27. Vernon, L. P.; Shaw, E. R.; Plant Physiol. 1969, 44, 1645.

28. Allen, J. F.; Holmes, N. G. Em Photosynthesis, Energy Transduction. A Practical Approach; Hipkinns, M. F.; Baker, N. R., eds.; IRL Press: Oxford, 1986.

29. Bussotti, F.; Strasser, R. A.; Schaub, M.; Environ. Pollut. 2007, 147, 430.

30. Srivastava, A.; Strasser, R. J.; J. Scient. Ind. Res. 1997, 56, 133.

31. Hernández-Terrones, M. G.; Aguilar, M. I.; King-Díaz, B.; LotinaHennsen, B. Pestic. Biochem. Physiol. 2003, 77, 12.

32. Demuner, A. J.; Barbosa, L. C. A.; Veiga, T. A. M.; Barreto, R. W.; KingDiaz, B.; Lotina-Hennsen, B.; Biochem. Syst. Ecol. 2006, 34, 790.

33. King-Díaz, B.; Castelo-Branco, P. A.; dos Santos, F. J. L.; Rubinger, M. M. M.; Ferreira-Alves, D. L.; Piló-Veloso, D.; Lotina-Hennsen, B.; Pestic. Biochem. Physiol. 2010, 96, 119.

34. Torres-Romero, D.; King-Díaz, B.; Strasser, R. J.; Jimenez, I. A.; LotinaHennsen, B.; Bazzocchi, I. L.; J. Agric. Food Chem. 2010, 58, 10847.

35. de Oliveira, D. M.; Aguilar, M. I.; King-Díaz, B.; Vieira-Filho, S. A.; Pains-Duarte, L.; Silva, G. D. F.; Lotina-Hennsen, B.; Molecules 2011, 16, 9939. 